\title{
Mapping the Yeast Host Cell Response to Recombinant Membrane Protein Production: Relieving the Biological Bottlenecks
}

\author{
Mark P Ashe ${ }^{1}$ and Roslyn M Bill ${ }^{2}$ \\ ${ }^{1}$ Faculty of Life Sciences, University of Manchester, Oxford Road, Manchester M13 9PT, UK \\ ${ }^{2}$ School of Life \& Health Sciences and Aston Research Centre for Healthy Ageing, Aston \\ University, Aston Triangle, Birmingham B4 7ET, UK
}

Keywords: yeast; translation; stress

\begin{abstract}
Author to whom all correspondence is to be sent: Roslyn M Bill, School of Life \& Health Sciences and Aston Research Centre for Healthy Ageing, Aston University, Aston Triangle, Birmingham B4 7ET, UK; +44 121204 4274; r.m.bill@aston.ac.uk
\end{abstract}




\begin{abstract}
Membrane proteins are targeted by over $50 \%$ of marketed pharmaceuticals, and as most membrane proteins are not naturally abundant, they must be produced recombinantly for the structural biology that is a pre-requisite to structure-based drug design. Unfortunately, obtaining high yields of functional, recombinant membrane proteins remains a major bottleneck in contemporary bioscience. Trial-and-error optimisation studies have not (and cannot) reveal mechanistic details of the biology of recombinant protein production, highlighting the need for defined and rational optimisation strategies based upon experimental observation. To this end, we have published a transcriptome and subsequent genetic analysis that has identified genes implicated in high-yielding yeast cell factories. These results have highlighted a role for alterations to a cell's protein synthetic capacity in the production of high yielding cells: paradoxically, reduced protein synthesis favors higher yields of recombinant membrane protein. These results highlight a potential bottleneck at the protein folding or translocation stage of protein production.
\end{abstract}




\section{Introduction}

Membrane proteins are the targets of well over $50 \%$ of pharmaceuticals on the market. In all cases these drugs have been designed in the absence of specific structural knowledge of their target. For example, G protein-coupled receptors (GPCRs) are the largest human receptor family controlling a range of essential processes and yet the structures of just six of them are available, five having been published only in the last 3 years [1, 2]. In 1997, 23 pharmaceuticals were approved by the FDA, with just 17 approvals in 2007 [3]. Furthermore, 418 potential biopharmaceutical medicines were in development in 2006 but only 24 new medicines were approved in 2008. This slow down is due in part to the lack of new structural and functional information available for membrane proteins [4]. Since they are not naturally abundant, membrane proteins must be overproduced for the detailed studies that will validate them for entry into the drug discovery pipeline. Consequently obtaining high yields of functional, recombinant membrane protein remains a major research challenge $[5,6]$.

We and others have recognized that the root of the problem is the host organism, and the lack of knowledge about the intricate cellular biology within. In our own work on yeast, we previously identified 39 Saccharomyces cerevisiae genes whose expression was significantly altered when the aquaporin, Fps1, was produced under high-yielding conditions $\left(20^{\circ} \mathrm{C}\right.$, pH5) compared to lowyielding standard growth conditions $\left(30^{\circ} \mathrm{C}, \mathrm{pH} 5\right)[7]$. In particular an essential gene, $B M S 1$, with a role in ribosome biogenesis, was identified as always up-regulated in high-yielding host cells [8]. This approach stands out from the repeated rounds of trial-and-error "optimisation" typically adopted in the field [9] as well as targeted deletion approaches in protease or secretion pathways, based on speculation of where bottlenecks might be. Although these approaches could be successful on a case-by-case basis, they have not (and cannot) reveal the mechanisms at work in a high-yielding cell. In contrast, genetic methods have enabled the engineering of high-yielding yeast strains.

The challenge now is to understand the underlying mechanisms, which will facilitate the reliable production of membrane proteins through an improved understanding of the yeast host cell. In the Wiley- ${ }^{3} \mathrm{CH}$ 
long-term it will contribute directly to the drug discovery pipeline and hence benefit an ageing population who will increasingly require access to new drugs to combat diseases including diabetes, cancer, and neurological disorders, as well as providing solutions for developing nations against diseases such as malaria and viral infection.

\section{The biosynthesis of recombinant membrane proteins}

Our current understanding of how native membrane proteins are synthesized in the cell is incomplete (Fig. 1), with each host cell having an array of molecules involved in the biogenesis pathway $[10,11]$. In principle any membrane protein could be produced recombinantly in any host cell system, but the yield is likely to be very low on account of these differences. Consequently, the preferred host cell is generally accepted to be that which is closest in origin to the source of the target membrane protein, which reflects the unknown requirements for a range of biosynthetic components. As this may not always be the most practical of solutions, especially for producing human membrane proteins, strategies are required that allow heterologous host cells to achieve the extended production profiles (often in excess of 2 hours) necessary for the biosynthesis of human membrane proteins $[12,13]$. Successful approaches have therefore typically relied on using low copy number plasmids, weak promoters and low temperatures as this is thought to allow sufficient time for folding of the membrane protein, whilst keeping the amount of mRNA encoding the mammalian membrane protein, and hence the stress on the host cell, to a minimum.

It is well known that membrane proteins can fold inefficiently. The cystic fibrosis transmembrane conductance regulator is a good example of this, as only $25-30 \%$ of the nascent polypeptide chain makes it to the cell surface in a correctly-folded state, with the remainder being misfolded and degraded in the endoplasmic reticulum (ER). One strategy to improve the functional yield of membrane proteins has therefore been to co-express molecular chaperones that are known to facilitate folding. One of the first examples of this relatively unexplored approach was performed by Chris Tate in 1999 [14] and gave a 3-fold improvement in functional yield of the serotonin transporter (SERT) in the baculovirus system. However, co-expression of further molecular 
chaperones actually decreased SERT yields, suggesting that the biosynthetic bottleneck had now been shifted to another step.

The more recent application of "omics" technologies has been driven by attempts to obtain a global understanding of recombinant membrane protein production in bacteria and yeast $[8,15]$ and to identify the mechanisms associated with high-yielding cells. In the prokaryote host, Escherichia coli, increases in levels of chaperones and proteases were found to be associated with increased membrane protein production [15], which concurred with the SERT experiments in the baculovirus system. The authors further speculated that low yields of recombinant membrane proteins might be due to limited Sec translocon capacity. A second prokaryote, the Gram-positive bacterium Lactococcus lactis, has also been used to produce a wide range of eukaryotic and prokaryotic membrane proteins [16], enabling a comparison of L. lactis and E. coli [17]. Although a large fraction of proteins could be produced in both hosts, some could only be produced in one or the other. Notably, for about half of the proteins produced in E. coli additional bands of lower molecular weight were observed, indicative of breakdown products, whereas only $10 \%$ of the proteins produced in L. lactis were degraded. A genetic study of L. lactis, identified a series of mutations specific to the L. lactis system that did not overlap with the findings in E. coli: three mutant strains that displayed 2- to 8-fold improvements in the yields of several target proteins were shown to carry single-site mutations in the nisK gene. NisK is the sensor protein of a two-component regulatory system that directs nisin-A-mediated expression [18].

In our own study of the $S$. cerevisiae response to membrane protein production [7], genes that were down-regulated under low yielding conditions were up-regulated under high-yielding conditions and vice versa [8] (Table 1). This lent confidence to the fact that these genes might have a role in high-yielding protein production, which was subsequently further validated by strain engineering. Specifically, we used this approach to engineer the first yeast production strains (GB0813253.2) by rational design [19]. In particular, the spt3 $\Delta$, srb5 $\Delta$ and $g c n 5 \Delta$ mutant strains were effective production hosts not only for the glycerol channel, Fps1, where the yield improvement was 10 - to 60 -fold higher than a wild-type control, but also the human GPCR, 
adenosine $2 \mathrm{a}$ receptor (hA2aR), where we doubled the functional yield compared to wild-type [8]. Although the three deleted genes have previously been identified as components of transcriptional complexes [20], improved yields of Fps1 were not correlated with increased promoter activity or FPS1 transcript copy number in the deletion strains. An observed up-regulation of BMS1 of 6- to 7fold over wild-type in each of these three deletion strains, as determined by Q-PCR, suggested the importance of ribosome biogenesis in high-yielding membrane protein experiments. Subsequent overexpression of BMS1, where the endogenous promoter is replaced with a doxycycline-titratable promoter in the genome, revealed that maximal Fps1 yield was significantly correlated with an optimum level of BMS1 transcript, which was further correlated with a changed ribosomal subunit stoichiometry. This observation was also true at different doxycycline concentrations for hA2aR and soluble GFP [8].

The knowledge generated by these and other studies should now provide an impetus to define the characteristics of high-yielding strains. Unsurprisingly, however, differences emerge between host cells. For example, our transcript analysis in yeast [7] identified up-regulation of SEC62 in high yielding protein production. Sec62 is an essential subunit of the Sec63 complex (Sec63, Sec62, Sec66 and Sec72) and with the Sec61 complex, Kar2 (the yeast binding immunoglubulin protein, BiP) and Lhs1 forms a channel competent for SRP-dependent and post-translational SRPindependent protein targeting and import into the ER. We found a SEC63 over-expression strain did not give improved recombinant protein yields. Additionally, we found that SRP102, which encodes the signal recognition particle receptor $\beta$ subunit, was down-regulated in high-yielding experiments and again that a srp102s strain gave only wild-type yields of membrane protein. Whilst this does not preclude the eukaryotic secretory pathway from having a limiting effect in $S$. cerevisiae translational efficiency, it does highlight clear differences between prokaryotic and eukaryotic cells. As discussed in the next section, some features of high-yielding cells are likely to be common to a range of host types, whilst many will no doubt turn out to be host- and/or target protein-specific. 


\section{The characteristics of high-yielding yeast cells}

\subsection{Slowed cell growth}

The slowing of growth that can accompany high-yielding protein production [8, 21] is often attributed to the metabolic burden associated with achieving these yields. Such observations are typical for cells which have improved "per cell" yields; more protein in the membrane of every cell equates to higher total yields. We have recently shown that such high-yielding yeast cells have a more efficient metabolism than low-yielding cells [8]. For $S$. cerevisiae, these high yielding conditions are associated with low fermentative activity [8]: $16 \%$ less ethanol and $6.5 \%$ less dry weight than wild-type under the same conditions and a lower average exponential phase $R Q(R Q$ = 3.6) than wild-type $(R Q=4.1$; in agreement with literature values for wild-type strains grown on glucose [22]). These observations are consistent with measurements of metabolic load in other recombinant host systems, albeit those producing heterologous soluble proteins: for example, Kemp and colleagues used on-line flow microcalorimetry data to examine metabolic load in mammalian cells [23], while thermal methods [24] and analysis of off-gases [21] were used to monitor E. coli cultures.

The accumulation of host cell biomass might be expected to provide a complementary strategy to increase the total protein yield; more cells equates to more membranes and hence to more recombinant protein. For example, the widely-used E. coli "Walker strains" (C41 and C43) apparently result in higher yields of recombinant membrane protein because their growth is not strongly inhibited upon induction. This results in increased biomass rather than increased "per cell" yields [25]. In yeast, however, the specific activity of some GPCRs is often lower when high cell density cultures are induced [26]. Indeed, it has been noted that higher cell densities can generate cellular stresses leading to modifications in membrane composition [27] and that this modified environment influences the activity of recombinant proteins. Consequently, medium cell density fermentation procedures for GPCR production have been suggested to be preferable to ones where biomass yields are maximized [26]. In a recent example of host development, a purely respiratory $S$. cerevisiae strain was reported that has improved biomass properties, permitting increased volumetric yields without the need to resort to complex control or cultivation schemes. In 
the case of the human adenosine $A_{2 A}$ receptor $\left(A_{2 A} R\right)$, the functional yield obtained in wild-type cells was quadrupled when using this strain under the same experimental conditions [28, 29].

\subsection{Reduced translational activity}

The finding that high-yielding yeast strains can have altered ribosomal subunit stoichiometry highlights the protein synthetic machinery and translational capacity of the cell as key determinants of improved recombinant membrane protein yield. As ribosome biogenesis is a major consumer of cellular energy resources and its regulation is intimately linked to cell size, which in turn affects cell growth as well as influencing other features such as the way that cells respond to stress, these observations are further consistent with the slow growth phenotype of high-yielding cells discussed in the previous section.

Previously, high yields of secreted recombinant protein have been obtained upon overexpression of $R P P O$, which encodes a ribosomal protein [30]. Furthermore, the production of heterologous membrane proteins has been shown to lead to the de-repression of GCN4 translation [31]. GCN4 serves as an intracellular marker for the level of the eIF2-GTP-Met-tRNAi ternary complex, which is critical for highly active translation initiation [32]. Overall, these results suggest that production of heterologous membrane proteins represses global cap-dependent translation initiation. For specific mRNAs, such as GCN4, translation is activated under conditions where the global translation initiation is inhibited via upstream open reading frames [33]. Another mechanism allowing the expression of specific mRNAs under conditions of global translation repression has more recently been described where the presence of adenosine-rich sequences upstream of the start codon leads to translation initiation via a cap-independent mechanism [34]. Intriguingly, the insertion of adenosine residues upstream of the start codon has also been identified as stimulating increased yields of heterologous proteins [35]. Thus, in attempting to rationally optimize heterologous membrane protein production, careful assessment of the rate and mechanism of synthesis of the heterologous protein relative to endogenous protein may prove critical. 


\subsection{An up-regulated unfolded protein response}

A key consideration when optimizing the production of recombinant proteins via the secretory pathway is the physiological responses that have evolved to maximize protein folding in the ER. The unfolded protein response (UPR) senses increases in unfolded protein within the ER and transmits this signal to pathways that increase the level of ER chaperones to maximize cellular output (Fig. 2). Indeed, yeast cells producing a GPCR were found to exhibit the hallmarks of a constitutively-active UPR [36]. In mammalian cells, activation of UPR-like responses has been shown to significantly improve recombinant protein production [37-39]. Similarly, in yeast overexpression of the genes encoding the Kar2 and PDI chaperones both reduced the UPR and increased heterologous protein production [40].

The UPR pathway is significantly simpler in yeast than in higher eukaryotes as only a single transmembrane protein kinase/nuclease, Ire1, is involved (Fig. 2). In current models, the accumulation of unfolded protein sequesters the Kar2 chaperone away from Ire1 causing it to dimerize and autophosphorylate. This activates the nuclease activity of Ire1 towards its cytoplasmic substrate Hac1 thereby inducing an unusual splicing event. This in turn leads to translation of the Hac1 transcription factor allowing the induction of several hundred genes, including ER-resident chaperones [41]. Clearly, as well as improving the yield of recombinant membrane proteins, many of the factors highlighted above, such as reduced protein synthesis, slower growth rate and lower cell densities, will also influence the level of UPR in cells. The UPR has therefore been identified as a target for improvements in heterologous protein yield in different yeast species [27], including $S$. cerevisiae [42], in order to produce recombinant membrane proteins. In the latter study, the UPR was exploited to optimize synthesis rates of membrane proteins in order to most efficiently use the cell's biosynthetic machinery. The authors noted, however, that some membrane proteins (especially those from yeast or plants) had little effect on the activity of the UPR, meaning that this may not be a globally applicable approach. Overexpression of $P$. pastoris HAC1 was subsequently used to increase the production of some heterologous proteins in P. pastoris, including the doubling of functional yields of a GPCR [43]. Overall, it is intriguing that in contrast to the mammalian UPR, the simpler UPR of yeast does not 
involve the down-regulation of translation to reduce the protein synthetic load on the ER [44]. It is therefore possible that a reduced protein synthetic capacity in yeast has proved particularly effective in improving recombinant protein yields since it targets an area that is unregulated in response to unfolded protein in cells.

\subsection{Up-regulation of BMS1}

Bms1, an essential nucleolar protein that is conserved throughout the eukaryotic kingdom, has a regulatory role in the biogenesis of the $40 \mathrm{~S}$ subunit [45] as well as being a GTP-binding protein [46]. We have shown that recombinant protein translation is clearly enhanced by the overexpression of BMS1 and that at maximal Fps1 yield there are elevated levels of both $40 \mathrm{~S}$ and 60 S subunits (Fig. 3) without any significant decrease in the levels of 80 S or polysome [8]. When the ribosome subunits were dissociated in the presence of EDTA, the ratio of 605 to $40 S$ subunits was 2:1 under the highest yielding conditions [8], but the relationship between this altered stoichiometry and improved recombinant protein yield remains unclear. Nonetheless, we are unaware of any other single effector that exerts such a huge effect in yeast or any other host cell.

Ribosomes catalyze protein synthesis in all cells, but in eukaryotic cells $>170$ proteins and $>70$ RNAs are involved in a complex and highly regulated process. Bms1 has been proposed to interact with Rcl1 in a GTP-dependent manner, shuttling Rcl1 to pre-ribosomes via its affinity for U3 snoRNA [47]. We find that overexpression of $R C L 1$ does not correlate with improved protein yields, but this may be due to Bms1 being limiting. Using a more severe FAL1 mutant strain [48], which has a massively increased 60S:40S ratio due to a severe reduction in $40 \mathrm{~S}$ subunit biogenesis, we observe no improvement in recombinant protein yield. Therefore the more subtle alterations in ribosomal stoichiometry of the BMS1 overexpression strain appear critical for high recombinant protein yields. We previously noted that mice that lacked $40 S$ synthesis, but had normal 605 synthesis in the liver, survived for several weeks. Their livers responded to fasting and re-feeding cycles, in which the mass of the liver nearly doubles. However, partial hepatectomy in normal animals led to rapid re-growth and cell division, but the livers of mice defective in the production of $40 \mathrm{~S}$ ribosomal subunits did not re-grow and showed no signs of cell division [49]. 
These results suggest that a lack of $40 \mathrm{~S}$ ribosome biogenesis can induce a checkpoint control that prevents cell cycle progression. This is consistent with our observed slowing of growth that accompanies high-yielding protein production.

\section{Concluding remarks}

We have shown that comparative transcriptome analysis of $S$. cerevisiae host cells during highversus low-yielding recombinant protein production experiments can guide strain engineering. In particular, by titrating the overexpression of $B M S 1$, a ribosome biogenesis gene, the functional yields of a range of membrane proteins can be improved. With the recent publication of a curated $P$. pastoris genome [50] it will now be possible to transfer this technology to a second industriallyrelevant yeast species and we further predict that this approach will be used for a range of host cells and protein targets [51]. As array techniques have improved, it is now possible to relate changes in mRNA with changes at the protein level. A comparative proteome analysis of membrane versus soluble protein production in $E$. coli highlighted that the cytoplasmic membrane protein translocation machinery might be limiting in low-yielding bacterial transformants [15]. Interestingly, we found no evidence for this in yeast [8], which highlights clear differences between prokaryotic and eukaryotic cells. With time, the ability to combine the outputs from these types of studies with those from metabolomic approaches will contribute to a true systems biotechnological description of recombinant membrane protein production in yeast.

\section{Acknowledgements}

This work was supported by the contracts LSHG-CT-2004-504601 (E-MeP), LSHG-CT-2006037793 (OptiCryst) and HEALTH-F4-2007-201924 (EDICT) to RMB, and Wellcome Trust (088141/Z/09/Z) and BBSRC (BB/G012571/1) research grants to MPA.

\section{References}

[1] Costanzi, S., Siegel, J., Tikhonova, I. G., Jacobson, K. A., Rhodopsin and the others: a historical perspective on structural studies of G protein-coupled receptors. Curr Pharm Des 2009, 15, 3994-4002.

[2] Sela, I., Golan, G., Strajbl, M., Rivenzon-Segal, D., et al., G protein coupled receptors -in silico drug discovery and design. Curr Top Med Chem 2010, 10, 638-656. 
[3] Rader, R. A., Paucity of Biopharma Approvals Raises Alarm; Lower Numbers, Novelty, and Economic Impact Indicate Problems. Genetic Engineering News 2008, 28.

[4] Bill, R. M., Henderson, P. J. F., Iwata, S., Kunji, E. R. S., et al., Widening bottlenecks in membrane protein structure pipelines. Nature Biotechnol 2011, in press.

[5] Graf, A., Dragosits, M., Gasser, B., Mattanovich, D., Yeast systems biotechnology for the production of heterologous proteins. FEMS Yeast Res 2009, 9, 335-348.

[6] Park, J. H., Lee, S. Y., Kim, T. Y., Kim, H. U., Application of systems biology for bioprocess development. Trends Biotechnol 2008, 26, 404-412.

[7] Bonander, N., Hedfalk, K., Larsson, C., Mostad, P., et al., Design of improved membrane protein production experiments: Quantitation of the host response. Protein Sci 2005, 14, 17291740.

[8] Bonander, N., Darby, R. A., Grgic, L., Bora, N., et al., Altering the ribosomal subunit ratio in yeast maximizes recombinant protein yield. Microb Cell Fact 2009, 8, 10.

[9] Islam, R. S., Tisi, D., Levy, M. S., Lye, G. J., Framework for the rapid optimization of soluble protein expression in Escherichia coli combining microscale experiments and statistical experimental design. Biotechnol Prog 2007, 23, 785-793.

[10] Bibi, E., Early targeting events during membrane protein biogenesis in Escherichia coli. Biochim Biophys Acta 2010.

[11] Wickner, W., Schekman, R., Protein translocation across biological membranes. Science 2005, 310, 1452-1456.

[12] Petaja-Repo, U. E., Hogue, M., Laperriere, A., Walker, P., Bouvier, M., Export from the endoplasmic reticulum represents the limiting step in the maturation and cell surface expression of the human delta opioid receptor. J Biol Chem 2000, 275, 13727-13736.

[13] Ward, C. L., Kopito, R. R., Intracellular turnover of cystic fibrosis transmembrane conductance regulator. Inefficient processing and rapid degradation of wild-type and mutant proteins. $J$ Biol Chem 1994, 269, 25710-25718.

[14] Tate, C. G., Whiteley, E., Betenbaugh, M. J., Molecular chaperones stimulate the functional expression of the cocaine-sensitive serotonin transporter. J Biol Chem 1999, 274, 17551-17558.

[15] Wagner, S., Baars, L., Ytterberg, A. J., Klussmeier, A., et al., Consequences of membrane protein overexpression in Escherichia coli. Mol Cell Proteomics 2007, 6, 1527-1550.

[16] Kunji, E. R., Slotboom, D. J., Poolman, B., Lactococcus lactis as host for overproduction of functional membrane proteins. Biochim Biophys Acta 2003, 1610, 97-108.

[17] Surade, S., Klein, M., Stolt-Bergner, P. C., Muenke, C., et al., Comparative analysis and "expression space" coverage of the production of prokaryotic membrane proteins for structural genomics. Protein Sci 2006, 15, 2178-2189.

[18] Linares, D. M., Geertsma, E. R., Poolman, B., Evolved Lactococcus lactis strains for enhanced expression of recombinant membrane proteins. J Mol Biol 2010, 401, 45-55.

[19] Sevastsyanovicha, Y., Alfasia, S., Cole, J., Recombinant protein production: a comparative view on host physiology New Biotechnology 2009, 25, 175-180

[20] Wu, P. Y. J., Ruhlmann, C., Winston, F., Schultz, P., Molecular architecture of the S. cerevisiae SAGA complex. Molecular Cell 2004, 15, 199-208.

[21] Hoffmann, F., Rinas, U., On-line estimation of the metabolic burden resulting from the synthesis of plasmid-encoded and heat-shock proteins by monitoring respiratory energy generation. Biotechnol Bioeng 2001, 76, 333-340.

[22] Barford, J. P., A general model for aerobic yeast growth: Batch growth. Biotechnol Bioeng 1990, 35, 907-920.

[23] Kidane, A., Guan, Y., Evans, P., Kaderbhai, M., Kemp, R., Comparison of heat flux in wild-type and genetically-engineered chinese hamster ovary cells. J Thermal Anal 1997, 49, 771-783.

[24] Gill, N. K., Appleton, M., Lye, G. J., Thermal profiling for parallel on-line monitoring of biomass growth in miniature stirred bioreactors. Biotechnol Lett 2008, 30, 1571-1575.

[25] Wagner, S., Klepsch, M. M., Schlegel, S., Appel, A., et al., Tuning Escherichia coli for membrane protein overexpression. Proc Natl Acad Sci U S A 2008, 105, 14371-14376.

[26] Singh, S., Gras, A., Fiez-Vandal, C., Ruprecht, J., et al., Large-scale functional expression of WT and truncated human adenosine A2A receptor in Pichia pastoris bioreactor cultures. Microb Cell Fact 2008, 7, 28.

[27] Mattanovich, D., Gasser, B., Hohenblum, H., Sauer, M., Stress in recombinant protein producing yeasts. J Biotechnol 2004, 113, 121-135. 
[28] Bonander, N., Ferndahl, C., Mostad, P., Wilks, M. D., et al., Transcriptome analysis of a respiratory Saccharomyces cerevisiae strain suggests the expression of its phenotype is glucose insensitive and predominantly controlled by Hap4, Cat8 and Mig1. BMC Genomics 2008, 9, 365. [29] Ferndahl, C., Bonander, N., Logez, C., Wagner, R., et al., Increasing cell biomass in Saccharomyces cerevisiae increases recombinant protein yield: the use of a respiratory strain as a microbial cell factory. Microb Cell Fact, 9, 47.

[30] Wentz, A. E., Shusta, E. V., Enhanced secretion of heterologous proteins from yeast by overexpression of ribosomal subunit RPP0. Biotechnol Prog 2008, 24, 748-756.

[31] Steffensen, L., Pedersen, P. A., Heterologous expression of membrane and soluble proteins derepresses GCN4 mRNA translation in the yeast Saccharomyces cerevisiae. Eukaryot Cell 2006, 5, 248-261.

[32] Pavitt, G. D., elF2B, a mediator of general and gene-specific translational control. Biochem Soc Trans 2005, 33, 1487-1492.

[33] Bourbon, H. M., Aguilera, A., Ansari, A. Z., Asturias, F. J., et al., A unified nomenclature for protein subunits of Mediator complexes linking transcriptional regulators to RNA polymerase II. Molecular Cell 2004, 14, 553-557.

[34] Gilbert, W. V., Zhou, K., Butler, T. K., Doudna, J. A., Cap-independent translation is required for starvation-induced differentiation in yeast. Science 2007, 317, 1224-1227.

[35] Krynetski, E. Y., Drutsa, V. L., Kovaleva, I. E., Luzikov, V. N., High yield expression of functionally active human liver CYP2D6 in yeast cells. Pharmacogenetics 1995, 5, 103-109.

[36] O'Malley, M. A., Mancini, J. D., Young, C. L., McCusker, E. C., et al., Progress toward heterologous expression of active G-protein-coupled receptors in Saccharomyces cerevisiae: Linking cellular stress response with translocation and trafficking. Protein Sci 2009, 18, 2356-2370. [37] Ohya, T., Hayashi, T., Kiyama, E., Nishii, H., et al., Improved production of recombinant human antithrombin III in Chinese hamster ovary cells by ATF4 overexpression. Biotechnol Bioeng 2008, 100, 317-324.

[38] Omasa, T., Takami, T., Ohya, T., Kiyama, E., et al., Overexpression of GADD34 enhances production of recombinant human antithrombin III in Chinese hamster ovary cells. J Biosci Bioeng 2008, 106, 568-573.

[39] Valkonen, M., Penttila, M., Saloheimo, M., Effects of inactivation and constitutive expression of the unfolded- protein response pathway on protein production in the yeast Saccharomyces cerevisiae. Appl Environ Microbiol 2003, 69, 2065-2072.

[40] Xu, P., Raden, D., Doyle, F. J., 3rd, Robinson, A. S., Analysis of unfolded protein response during single-chain antibody expression in Saccaromyces cerevisiae reveals different roles for BiP and PDI in folding. Metab Eng 2005, 7, 269-279.

[41] Mori, K., Signalling pathways in the unfolded protein response: development from yeast to mammals. J Biochem 2009, 146, 743-750.

[42] Griffith, D. A., Delipala, C., Leadsham, J., Jarvis, S. M., Oesterhelt, D., A novel yeast expression system for the overproduction of quality-controlled membrane proteins. FEBS Lett 2003, 553, 45-50.

[43] Guerfal, M., Ryckaert, S., Jacobs, P. P., Ameloot, P., et al., The HAC1 gene from Pichia pastoris: characterization and effect of its overexpression on the production of secreted, surface displayed and membrane proteins. Microb Cell Fact, 9, 49.

[44] Patil, C., Walter, P., Intracellular signaling from the endoplasmic reticulum to the nucleus: the unfolded protein response in yeast and mammals. Curr Opin Cell Biol 2001, 13, 349-355.

[45] Wegierski, T., Billy, E., Nasr, F., Filipowicz, W., Bms1p, a G-domain-containing protein, associates with Rcl1p and is required for 18S rRNA biogenesis in yeast. RNA 2001, 7, 1254-1267.

[46] Gelperin, D., Horton, L., Beckman, J., Hensold, J., Lemmon, S. K., Bms1p, a novel GTPbinding protein, and the related Tsr1p are required for distinct steps of $40 \mathrm{~S}$ ribosome biogenesis in yeast. RNA 2001, 7, 1268-1283.

[47] Karbstein, K., Doudna, J. A., GTP-dependent formation of a ribonucleoprotein subcomplex required for ribosome biogenesis. J Mol Biol 2006, 356, 432-443.

[48] Holmes, L. E., Campbell, S. G., De Long, S. K., Sachs, A. B., Ashe, M. P., Loss of translational control in yeast compromised for the major mRNA decay pathway. Mol Cell Biol 2004, 24, 2998-3010.

[49] Volarevic, S., Stewart, M. J., Ledermann, B., Zilberman, F., et al., Proliferation, but not growth, blocked by conditional deletion of 40 S ribosomal protein S6. Science $2000,288,2045-2047$. 
[50] De Schutter, K., Lin, Y. C., Tiels, P., Van Hecke, A., et al., Genome sequence of the recombinant protein production host Pichia pastoris. Nature Biotechnol 2009, 27, 561-566.

[51] Gasser, B., Sauer, M., Maurer, M., Stadlmayr, G., Mattanovich, D., Transcriptomics-based identification of novel factors enhancing heterologous protein secretion in yeasts. Appl Environ Microbiol 2007, 73, 6499-6507.

[52] Bonander, N., Bill, R. M., Relieving the first bottleneck in the drug discovery pipeline: using array technologies to rationalize membrane protein production. Expert Rev Proteomics 2009, 6, 501-505. 
Table

Table 1 Array data from a transcriptome analysis of yeast strains giving high or low yields of recombinant membrane protein: results for verified genes Genes were tabulated if their expression was changed when comparing both of the low yielding conditions (I and II) with normal growth conditions: $30^{\circ} \mathrm{C}(\mathrm{pH} 5)$ to $35^{\circ} \mathrm{C}(\mathrm{pH} 7)$ and $30{ }^{\circ} \mathrm{C}(\mathrm{pH} 5)$ to $35^{\circ} \mathrm{C}(\mathrm{pH} 5)$. The change is expressed as a factor (in parentheses), where that factor is $\mathrm{x}$ when a gene expressed with intensity 1 at $30{ }^{\circ} \mathrm{C} \mathrm{pH} 5$ is expressed with intensity $\mathrm{x}$ at the new condition. Arrows show whether the gene is up- or down-regulated. The results for the high-yielding conditions are shown for comparison. Only the data for ERG9 deviate from the pattern that genes that are down-regulated under low yielding conditions are up-regulated under high-yielding conditions and vice versa [52].

\begin{tabular}{|c|c|c|c|c|}
\hline $\begin{array}{l}\text { Systematic } \\
\text { name }\end{array}$ & Gene & $\begin{array}{c}30 \stackrel{\circ}{\circ} \mathrm{pH} 5 \\
\text { to } \\
35 \stackrel{\circ}{\mathrm{C}} \mathrm{pH} 7 \\
\text { (low yield I) }\end{array}$ & $\begin{array}{c}30 \stackrel{\circ}{\circ} \mathrm{pH} 5 \\
\text { to } \\
35 \stackrel{\circ}{\mathrm{C}} \mathrm{pH} 5 \\
\text { (low yield II) }\end{array}$ & $\begin{array}{c}30 \stackrel{\circ}{ }{ }^{\circ} \mathrm{pH} 5 \\
\text { to } \\
20^{\circ} \mathrm{C} \mathrm{pH} 5 \\
\text { (high yield) }\end{array}$ \\
\hline YPL217C & $B M S 1$ & $\Downarrow(0.2)$ & $\Downarrow(0.2)$ & $\Uparrow(2.0)$ \\
\hline YGR286C & $\mathrm{BlO2}$ & $\Uparrow(5.3)$ & $\Uparrow(5.5)$ & $\Downarrow(0.4)$ \\
\hline YPR019W & $C D C 54$ & $\Downarrow(0.2)$ & $\Downarrow(0.2)$ & $\Uparrow(2.6)$ \\
\hline YPL254W & $H F / 1$ & $\Downarrow(0.2)$ & $\Downarrow(0.1)$ & $\Uparrow(2.2)$ \\
\hline YBR296C & PHO89 & $\Downarrow(0.1)$ & $\Downarrow(0.04)$ & $\Uparrow(3.7)$ \\
\hline YBR253W & SRB6 & $\Downarrow(0.3)$ & $\Downarrow(0.3)$ & $\Uparrow(1.4)$ \\
\hline YBR288C & APM3 & $\Downarrow(0.2)$ & $\Downarrow(0.3)$ & $\Uparrow(1.7)$ \\
\hline YGL029W & CGR1 & $\Downarrow(0.2)$ & $\Downarrow(0.3)$ & $\Uparrow(1.7)$ \\
\hline YOR303W & CPA1 & $\Downarrow(0.3)$ & $\Downarrow(0.3)$ & $\Uparrow(1.6)$ \\
\hline YHR190W & $E R G 9$ & $\Uparrow(3.3)$ & $\Uparrow(3.0)$ & $\Uparrow(2.4)$ \\
\hline YPL187W & $m f(a l p h a) 1$ & $\Uparrow(3.9)$ & $\Uparrow(3.7)$ & $\Downarrow(0.6)$ \\
\hline YPL104W & MSD1 & $\Downarrow(0.3)$ & $\Downarrow(0.3)$ & $\Uparrow(1.5)$ \\
\hline YPL206C & $P G C 1$ & $\Downarrow(0.4)$ & $\Downarrow(0.4)$ & $\Uparrow(1.4)$ \\
\hline YFL036W & RPO41 & $\Downarrow(0.3)$ & $\Downarrow(0.3)$ & $\Uparrow(2.6)$ \\
\hline YPL094C & SEC62 & $\Downarrow(0.3)$ & $\Downarrow(0.2)$ & $\Uparrow(1.5)$ \\
\hline
\end{tabular}




\section{Figure legends}

Figure 1 Strategies to overcome bottlenecks in the production of recombinant membrane proteins. The scheme shows the pathway of membrane protein production in yeast cells. Various steps are depicted including mRNA transcription and processing in the nucleus, mRNA export into the cytoplasm through the nuclear pore, the synthesis of protein from mRNA, the translocation and insertion of protein into the endoplasmic reticulum (ER) membrane and the folding and glycosylation of membrane proteins through the secretory pathway. Strategies used to optimize recombinant membrane protein production are highlighted.

Figure 2 The yeast unfolded protein response (UPR). The scheme shows the consequences of unfolded protein accumulation in the endoplasmic reticulum (ER) lumen and the pathway that is consequently activated. Steps depicted include the dissociation of Kar2 from the Ire1 transmembrane kinase/endonuclease, the dimerisation and phosphorylation of Ire1, the unusual mechanism of HAC1 mRNA splicing and the role of Hac1 protein in the transcriptional activation of genes which increase the capacity of the ER.

Figure 3 Polysome profiles for the BMS1 and wild-type strains (A) Polysome $\mathrm{OD}_{254}$ profiles for yeast strains producing Fps1 in the absence (upper panels) or presence (lower panels) of 0.5 $\mu \mathrm{g} / \mathrm{mL}$ doxycycline. Addition of $0.5 \mu \mathrm{g} / \mathrm{mL}$ doxycycline to the $B M S 1$ mutant strain leads to maximum yields of Fps1 (70 times higher than wild-type) compared to low yields under all other conditions shown. Polysome peaks containing 2, 3 and 4 ribosomes are visible. (B) Ribosome disassociation profiles at $\mathrm{OD}_{254}$ for $50 \mathrm{mM}$ EDTA-treated samples of wild-type and BMS1 strains producing Fps1 in the absence and presence of $0.5 \mu \mathrm{g} / \mathrm{mL}$ doxycycline (upper and lower panels respectively). Changes in peak heights and ratios of 605 and 405 subunits for all four conditions are tabulated. Reproduced from reference [8]. 


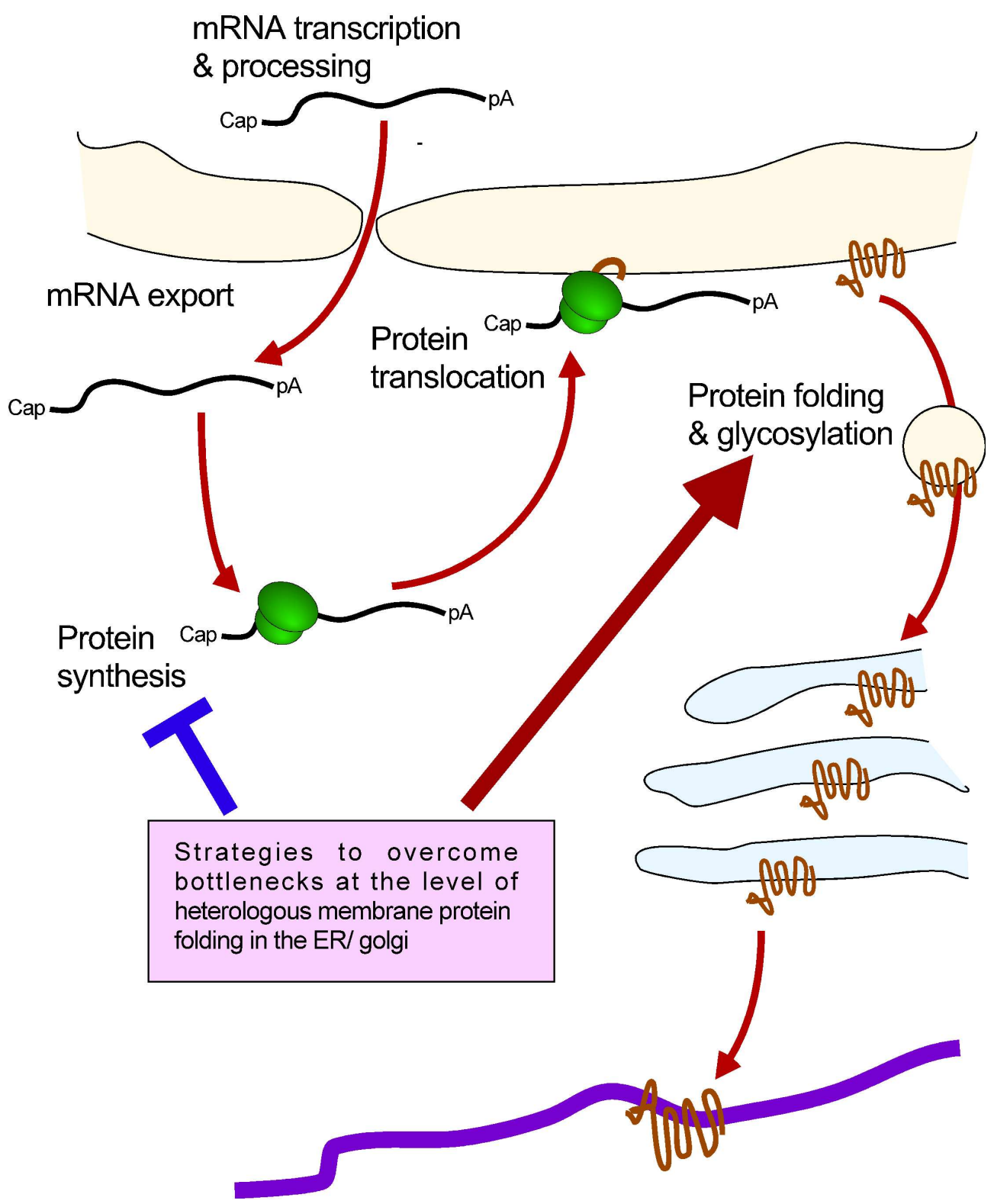




\section{Unable to Convert Image \\ The dimensions of this image (in pixels) are too large to be converted. For this image to convert, the total number of pixels (height $x$ width) must be less than 40,000,000 (40 megapixels).}


(A) Wild Type

BMS1 Mutant
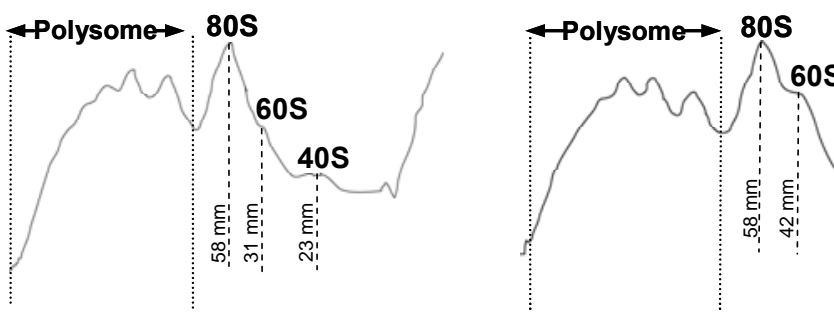

$40 \mathrm{~S} \quad 0 \mu \mathrm{g} / \mathrm{mL}$
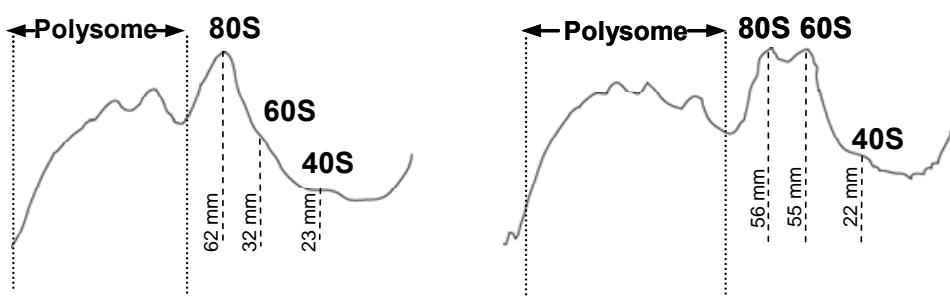

$0.5 \mu \mathrm{g} / \mathrm{mL}$ doxycycline

(B) Wild Type BMS1 Mutant

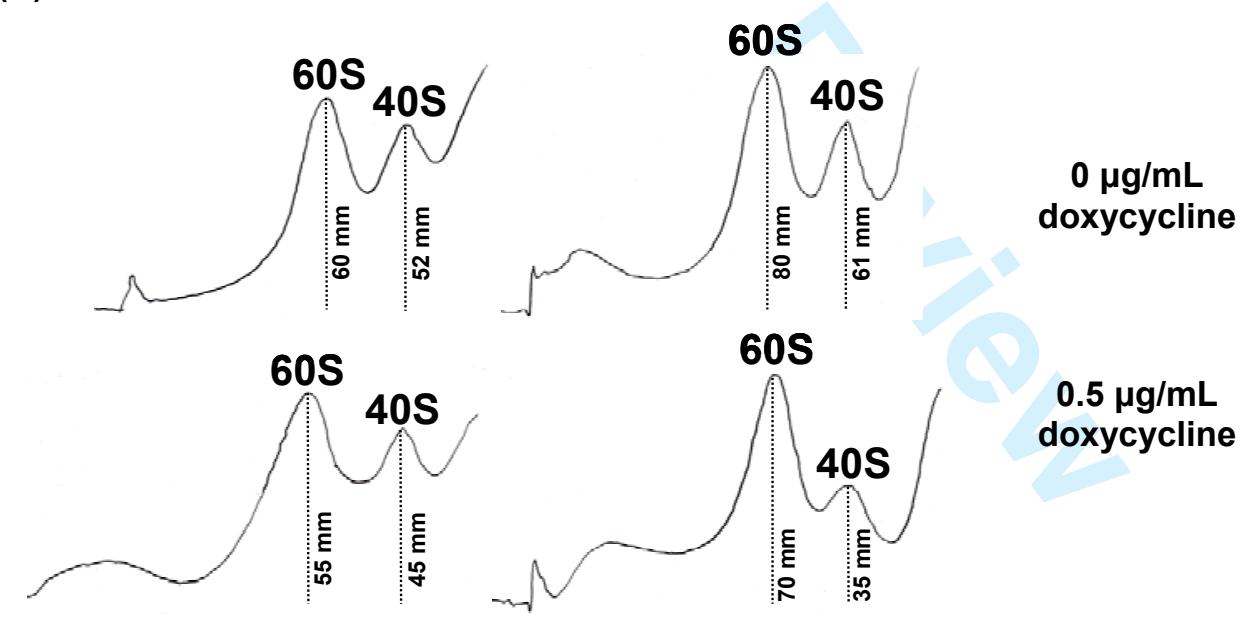

\begin{tabular}{l|ll||l} 
Change in peak heights $(\%)$ between culture conditions & $\mathbf{4 0 S}$ & $\mathbf{6 0 S}$ & $\begin{array}{l}\text { Change in } \\
\mathbf{6 0 S : 4 0 S}\end{array}$ \\
\hline WT to WT + doxycycline & -13.5 & -8.3 & No change \\
WT to yTHCBMS1 & +17.3 & +33.3 & No change \\
WT to yTHCBMS1 + doxycycline & -32.7 & +16.7 & 1.2 to 2.0 \\
yTHCBMS1 to yTHCBMS1 + doxycycline & -42.6 & -12.5 & 1.3 to 2.0
\end{tabular}

